\title{
INTEGRATED METHODS FOR PERFORMANCE MEASUREMENT IN ENTITIES FROM THE WINE SECTOR IN ROMANIA
}

\author{
Sorin Briciul \\ Dan Ioan Topor ${ }^{2}$ \\ Sorinel Căpuşneanu ${ }^{3}$
}

\begin{abstract}
This article highlights the authors attempt to identify modern cost calculation methods and appropriate performance measurement tools and their integration in order to improve the performance of the economic entities from the wine sector in Romania. Identifying methods and tools for performance measurement are based on an analysis of a representative sample of specialists from the economic entities from wine sector in Romania. The article is littered with empirical studies designed to highlight the performance of the economic entities from the wine sector based on using the integrated methods such as Activity Based Costing (ABC) method and Balance Scorecard (BS) method. All the results obtained based on the specific methodology of the methods studied are analyzed by the authors. The article ends with the conclusions of the authors on using integrated methods for measuring performance in the entities from the wine sector in Romania.
\end{abstract}

Keywords: Activity-Based Costing, Balanced Scorecard, performance, strategic managerial accounting, non financial indicators

JEL Codes: M41, M21

\section{Introduction}

The acceleration of the globalization process, the economical and financial crisis, the speed of the technological development, the companies' partnerships and mergers, the disappearance of the organization borders or the development of digital economy are just a few of the main causes which imposed the transformation of managerial accounting in order to adapt the techniques and its specific methods to the new information demands of the entity and company management. The continuous evolution of managerial accounting for the improvement of performance management methods and identifying new dimensions of type cost information related to decision making process and determining the performance of economic entities constituted the premise for choosing this research. Among the objectives taken in developing our study we can mention: identifying posibilities to improve the performances of economic entities from the wine sector by applying integrated methods specific to strategic managerial accounting such as Activity Based Costing method (ABC) and Balanced Scorecard method (BS) and their impact created by using them.The choice of instruments for measuring the performance of economic entities is a current topic in the light of the importance of attributes in the speed and sustainability of decision making by management, whose mission is vital to ensure the success of the business conducted by them.

\footnotetext{
1 “1 Decembrie 1918” University, Alba Iulia, Romania, e-mail: sorinbriciu@ gmail.com

2 “1 Decembrie 1918” University, Alba Iulia, Romania, e-mail: dan.topor@yahoo.com

3 “Dimitrie Cantemir" Christian University, Bucharest, Romania, e-mail: sorinelcapusneanu@ucdc.ro
} 


\section{Literature review}

In the late 1990s it was considered that in the future, managerial accounting will be developed in areas which involve a broad spectrum of interrelated disciplines such as performance management, asset management, environmental management, financial management, intellectual capital management, knowledge management, quality management and strategic management (Sharma, 1998). Reality has confirmed this through the appearance of managerial accounting for environmental management, the concept of corporate social responsibility, development of Activity Based Costing method (ABC), Activity Based Management (ABM), Balance Scorecard method (BS), Life-Cycle Costing method, Target Costing method (TC) and Strategic Managerial Accounting (SMA) in order to develop financial and non financial indicators which must allow the evaluation of intellectual capital or quality. The directions of managerial accounting were focused to integrate it into the risk management strategies, to anticipate the needs of performance and financial management and to finding the most efficient ways to assess the tangible resources and especially intangible of the organization.

Use of non-financial indicators combined with financial indicators of traditional management accounting methods has become a general practice recommended and accepted worldwide. Nonfinancial indicators began to be studied by numerous specialists 30-35 years ago and were developed in the 1990s (Anthony, Dearden and Vancil, 1976; Parker, 1979; Merchant, 1985; Schoenfeld, 1986; Eccles, 1991).

The relationship between resource planning process and performance measurement was developed through Pyramid Performance creating a new vision and strategies for measuring lower levels of both the financial indicators and non-financial indicators (Mc Nair et. al, 1990). All this resulted in a strategic measurement system which is Balance Scorecard (BS).

Balanced Scorecard was developed extensively in USA but in Europe too, especially in France was the dashboard was mostly studied and applied. Some specialists agree with the theory that, balanced scorecard was developed based on traditional French dashboard (Chiapello and Lebas, 1996), and other specialists have conducted comparative studies between the dashboard and balanced scorecard which have shown arguments for and against of two performance measurement tools, including their relation to socio-economic context of the country which they applied (Epstein and Manzoni, 1997; Mendoza and Zrihen, 1999).

The most active promoters of Balanced Scorecard were from USA (Kaplan and Norton, 1992, 1996, 1997). Other specialists have published their studies reporting the conditions of implementation and the advantages or disadvantages of Balanced Scorecard (Butler et. al., 1997; Frigo and Krumwiede, 2000). At the moment of publication, in 1992 the method wanted to give a concrete way of performance evaluation and integration of their management in the strategic management of organizations. Four years later the promoters of method, Kaplan and Norton propose to consider it as a performance management system and publish the first book that explains the practical aspects of implementing the method.

At the appearence of the second publication dedicated to Balances Scorecart method in 2000, the authors brought to the attention of theoreticians and practitioners another aspect of the methodthat of strategic management and control tool. The same authors introduce in the year 2004 the concept of strategic map which support the importance of the method in strategic management process previously explained and direct ways to implement it.

2008 was another important moment in the development of Balanced Scorecard, Kaplan and Norton arguing how instruments specific to the method lead to the alignment of strategy with operational area of activities. Also, the components of the method contained in the dashboard are the central element when the strategy turns into action.

From the historical point of view, the emergence of $A B C$ was marked by various events, such as the '60s period characterized by a high volume production and low range products, which led to application of the methods known to be traditional type. 
Also in 1960, accountants from General Electricare were facing a high volume of indirect costs, and for the first time they used the concept of activity just to describe the expenses caused by the stages of production, inventing a system which was based on activities for identifying costs. Years '70 and ' 80 were characterized through reduction of industrial superiority of the United States of America.

This was due to the need to develop new procedures for calculating costs, while the indirect costs gained a bigger role in the cost of production at the expense of direct costs. Knowledge and proper allocation of indirect costs was completed by the analysis of the causes that determined their volume. In the late '80s, C.A.M.I. (Consortium of Advanced Manufacturers International) first defined $\mathrm{ABC}$ as a method for identifying the causes of the connections between the activities cost and the cost drivers by measuring the process costs which activities referres to and cost objects.At this stage of development of managerial accounting, Activity-Based Costing method itself was known as a strategic initiative of the costing in business and is considered by experts an innovation in management costs. ABC identifies its origin in the U.S. in the late 80s, in "The hidden factory" paper (Miller and Vollmann, 1985). ABC promoters undergone a critical study the areas and places of common costs (indirect), and concluded that the decisive step for controlling indirect costs is to develop a model to detail and structure the causes the costs (Ebbeken et. al, 2000). Causes of activity cost calculation have their origin in changes in strategic positions of the entities, changes in demand of information for management, weaknesses in traditional cost calculation systems

The first attempt to construct a system of activity-based costing is attributed to American specialists Kaplan, Cooper and Johnson. Their efforts were limited to the transposition of direct tools of the reference with double function, elaborated and developed later in the plan cost calculation for production sectors on auxiliary sectors and places. In this direction others specialists have expressed similar concerns on the design and implementation of ABC (Porter, 1985; Lorino, 1997; Lebas, Mevellec 1999). According to studies of managerial accounting professionals, ABC method helps to refine the cost system by identifying individually activities as a foundation of objective cost (Horngren et al., 2009). ABC method was one of the challenges that cost calculation was exposed when it was felt the need to eliminate waste in all areas that generate expenses.This method refuses imputations in cascade and seeks to preserve the relationship between costs and causes that triggered (Bouquin, 2004). In European context ABC method consists in placing between responsability centers and products a intermediate category: calculation activity on activity costing offered by different centers and determining the proportion in which this products uses this type of activities for obtaining a much more reliable evaluation of products costs (Tabără and Briciu, 2012). Very inportant are the research made on budgeting and development line of some systems based on the responsability centers related to managerial accounting (Răchişan et. al, 2010; Groşanu et. al, 2009). Empirical research conducted on the effectiveness of the ABC method highlighted benefits by supporting the ongoing improvement process by developing methods for determining actual costs by increasing financial performance.

\section{Research methodology}

\section{Research questions}

To achieve the main objective of our scientific approach respectively identifying opportunities to improve the performance of the wine industry, we used a empirical study at a economic entity in the field, by applying integrated methods belonging to strategic managerial accounting. With other words we tried to find answers to the next questions:

1. What would be the most viable and effective performance measurement integrated methods for entities from wine industry in Romania?

2. Which are the advantages offered by the integrated methods for measuring performances?

3. Which would be the impact created by using this tools on entities from the wine sector in Romania? 


\section{Instrumentation}

This study was done by observing principles and specific rules of mixed research methodology. Research techniques and procedures used were: review of the literature, the use of various information sources, gathering and processing of data, summarizing the theoretical and research results and the questionnaire. This latest research technique we used to realize the empirical work of the paper. Questionnaire was used for the empirical part of our study and was structured to meet the objectives pursued and finding answers to research questions released. Questionnaires were sent electronically to 42 respondents situated on different hierarchical levels of an economic entity from wine sector, as follows: upper management 9 and 33 specialists in various departments. After collecting the questionnaires and performance data centralization, situation is as follows (tabel 1):

Table no.1.

Situation of categories of respondents

\begin{tabular}{|c|c|c|c|c|}
\hline \multirow{3}{*}{ Questions/Answers } & \multicolumn{4}{|c|}{ Category of respondents } \\
\hline & \multicolumn{2}{|c|}{ Management } & \multicolumn{2}{|c|}{$\begin{array}{l}\text { Specialists from } \\
\text { departments }\end{array}$} \\
\hline & for & against & for & against \\
\hline $\begin{array}{l}\text { 1. What would be the most viable and effective performance measurement } \\
\text { integrated methods for entities from wine industry in Romania? }\end{array}$ & & & & \\
\hline a) Standard-Cost method & $44,44 \%$ & $55,56 \%$ & $33,33 \%$ & $66,67 \%$ \\
\hline b) Activity-Based Costing method & $88,89 \%$ & $11,11 \%$ & $75,00 \%$ & $25,00 \%$ \\
\hline c) Balanced Scorecard method & $77,78 \%$ & $22,22 \%$ & $62,50 \%$ & $37,50 \%$ \\
\hline $\begin{array}{l}\text { 2. Which are the advantages offered by the integrated methods for measuring } \\
\text { performances? }\end{array}$ & & & & \\
\hline a) simplicity, efficiency and reduced calculation time & $50,00 \%$ & $50,00 \%$ & $54,16 \%$ & $45,83 \%$ \\
\hline b) reduced costs andreduced calculation time & $55,56 \%$ & $44,44 \%$ & $50,00 \%$ & $50,00 \%$ \\
\hline c) efficiency, reduced costs, profit maximization & $83,33 \%$ & $16,67 \%$ & $83,33 \%$ & $16,67 \%$ \\
\hline $\begin{array}{l}\text { 3. Which would be the impact created by using this tools on entities from the wine } \\
\text { sector in Romania? }\end{array}$ & & & & \\
\hline a) reducing costs & $66,67 \%$ & $33,33 \%$ & $66,67 \%$ & $33,33 \%$ \\
\hline b) gradual improvement in the results of the entity & $88,89 \%$ & $11,11 \%$ & $83,33 \%$ & $16,67 \%$ \\
\hline e) profit maximization & $77,78 \%$ & $22,22 \%$ & $62,50 \%$ & $37,50 \%$ \\
\hline
\end{tabular}

In terms of graphic (Figure 1) the situation is like this:

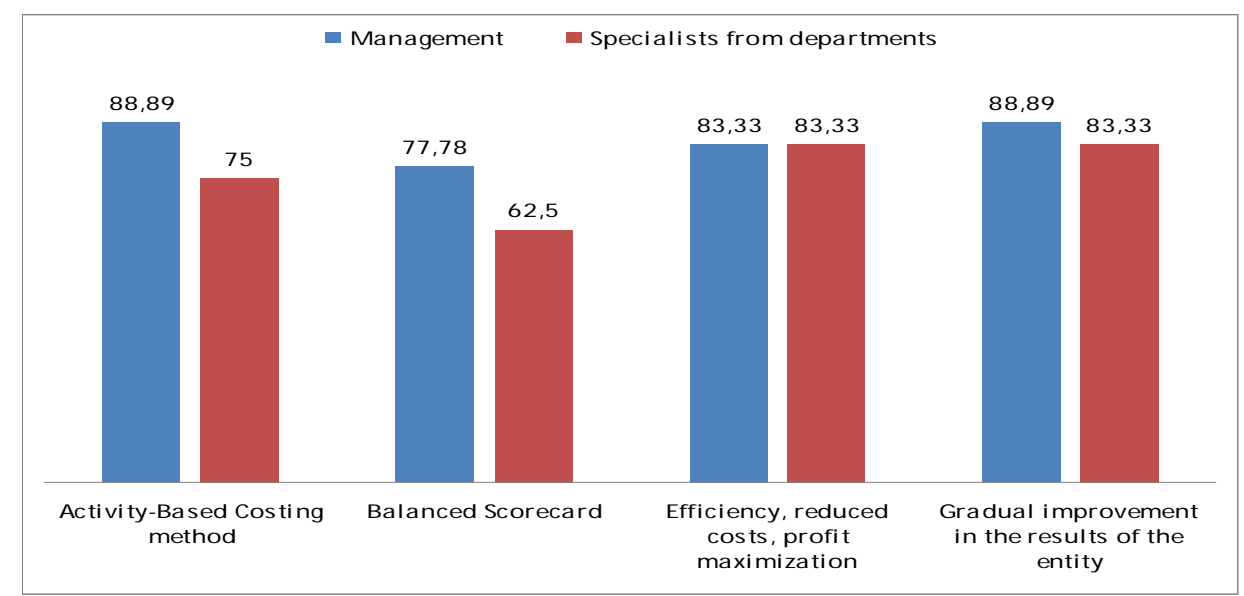

Figure no. 1. - Graphic representation of the responses of people interviewed

As it can be seen, the largest share in both categories surveyed on three questions pursued in our study advocated for use of Activity-Based Costing and Balanced Scorecard as effective and viable tools in offering efficiency, lower costs and contribute to the gradual improvement of the results of the entity. 


\section{Romania \\ Integrated tools for performance measurement in entities from the wine sector in}

Activity Based-Costing method

Using the $\mathrm{ABC}$ approach involves initially rearranging the organizational structure in a transversal way, as a set of processes that cuts an organization and crosses all the responsability centers and the hierarchical levels. ABC method "pilotage element of costs and transverse processes performance gives readable representation of the activities of operational officers (Demeestère, 2004), being based on an idea of improvement. Reduction of hierarchical levels and better coordination of actions are influenced by the reorganization objectives, namely the adoption of the three levels of transversal analysis (the processes, activities, and operations).

$\mathrm{ABC}$ calculation according to the specific entities from the wine sector, in a simplified form, involves the following steps:

1. Establishing the hierarchy of processes and identifying the main activities (auxiliary);

2. Establishing the cost drivers and building quantitative structures;

3. Allocation of costs indirectly to products based on specific drivers;

4. The calculation of the production cost

For the implementation of the $\mathrm{ABC}$ method is necessary to divide the production process in its component activities. This division, however, must be made in such a way that a balance between information needs and the cost of obtaining and processing such information must exist. We consider two of the models for the production of wine from the point of view of manufacturing phases (Figure 2).

\begin{tabular}{|c|c|c|}
\hline \multicolumn{3}{|c|}{ Processes } \\
\hline & Grape collection & \\
\hline & 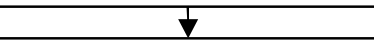 & \\
\hline & Wine production & \\
\hline & $\downarrow$ & \\
\hline & Processes for dissolution & \\
\hline \multicolumn{3}{|c|}{ Main activities } \\
\hline Model 1 (3 phases) & \multicolumn{2}{|r|}{ Model 2 (4 phases) } \\
\hline Pre-fermentation & & Grape processing \\
\hline$\downarrow$ & & $\downarrow$ \\
\hline Fermentation & & Fermentation \\
\hline$\downarrow$ & & $\nabla$ \\
\hline \multirow[t]{3}{*}{ Post-fermentation } & & Conditioning of wine \\
\hline & & $\downarrow$ \\
\hline & & Bottling \\
\hline \multicolumn{3}{|c|}{ Auxiliary activities } \\
\hline Using tanks and barrels & & \\
\hline \multicolumn{3}{|l|}{$\downarrow$} \\
\hline \multicolumn{3}{|l|}{ Using pumping equipment } \\
\hline \multicolumn{3}{|l|}{$\downarrow$} \\
\hline \multicolumn{3}{|l|}{ Using refrigeration equipment } \\
\hline \multicolumn{3}{|l|}{ 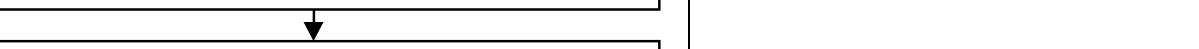 } \\
\hline Using equipment for cleaning and disinfecting & & \\
\hline
\end{tabular}

Figure no. 2. - Identification of the processes and activities 
Table 2 shows the composition of the costs of auxiliary activities.

Table no.2.

Composition of the costs of auxiliary activities

\begin{tabular}{|c|l|l|}
\hline Costs clasification & \multicolumn{1}{|c|}{ Content } & \multicolumn{1}{|c|}{ Contained costs } \\
\hline Basis equipment & $\begin{array}{l}\text { - tanks, pumps, cooling installations, } \\
\text { machinery }\end{array}$ & $\begin{array}{l}\text { - maintenance and operating } \\
\text { costs, depreciation }\end{array}$ \\
\hline Auxiliary equipment & - valves, hoses, taps & - annual cost of replacement \\
\hline $\begin{array}{c}\text { Other costs asociated to } \\
\text { the auxiliary activities }\end{array}$ & $\begin{array}{l}\text { - water, chemicals for cleaning, } \\
\text { electricity, preparation of activities, } \\
\text { quality control }\end{array}$ & - acquisition cost \\
\hline Indirect costs & $\begin{array}{l}\text { - cleaning tanks and barrels after use, } \\
\text { maintenance of cooling systems between } \\
\text { two uses }\end{array}$ & - labor price \\
\hline
\end{tabular}

Source: own systematization

An important step in applying $\mathrm{ABC}$ method is the identification of the asociated costs and the cost drivers. At the end of each activity, the wine cost from a certain phase is composed from the amount of grape, mash and wine from a previous stage at which is added the costs of different activities made for processing.

Regarding the costs of each activity, they can be grouped into direct costs and specific costs necessary to conduct the activity.

The direct costs will include labor costs concerning manufacturing or control its consumption of auxiliary materials (sulfur dioxide, bentonite etc.) the cost of specific equipment use and maintenance, equipment depreciation costs, energy and water costs and other costs directly identifiable as being specific analyzed activities. Additional or auxiliary costs include the costs of auxiliary activities (pumping, cooling, storage, etc.) that are needed to run the main activity. Table 3 presents a summary of the processes-activities-related costs-cost drivers' scheme that will be further developed in the case study by allocating appropriate monetary values.

Table no. 3 .

Processes-activities-related costs - cost drivers scheme

\begin{tabular}{|c|c|c|c|}
\hline Processes & Activities & Related costs & Cost drivers \\
\hline \multirow{3}{*}{$\begin{array}{l}\text { Collecting } \\
\text { grapes, } \\
\text { reception and } \\
\text { quality testing }\end{array}$} & $\begin{array}{l}\text { Selecting grapes, collecting and } \\
\text { transport }\end{array}$ & $\begin{array}{l}\text { Direct costs: the cost of grapes, labor } \\
\text { hours price, transport activities cost }\end{array}$ & \multirow{3}{*}{$\begin{array}{l}\text { Kilograms of } \\
\text { grapes }\end{array}$} \\
\hline & $\begin{array}{l}\text { Grapes reception (quality testing, } \\
\text { weighing, discharging) }\end{array}$ & $\begin{array}{l}\text { Direct costs: labor hour price, } \\
\text { depreciation of equipment, energy }\end{array}$ & \\
\hline & Related activities & $\begin{array}{l}\text { Auxiliary costs: the cost of ancillary } \\
\text { materials (shovels, buckets etc.) }\end{array}$ & \\
\hline \multirow{4}{*}{$\begin{array}{c}\text { Wine } \\
\text { production }\end{array}$} & $\begin{array}{l}\text { Activities before fermentation: } \\
\text { - destemming } \\
\text { - crushing } \\
\text { - Pumping in fermentation tanks }\end{array}$ & \multirow{4}{*}{$\begin{array}{l}\text { Direct costs: labor hour price, raw } \\
\text { material cost (previously calculated), } \\
\text { costs associated of equipment } \\
\text { (depreciation, maintenance, cleaning), } \\
\text { electricity } \\
\text { Indirect costs associated auxiliary } \\
\text { activities: preparation equipment }\end{array}$} & \multirow{4}{*}{$\begin{array}{l}\text { No. of days } \\
\text { No. of types of } \\
\text { wine produced } \\
\text { The amount of } \\
\text { wine on types of } \\
\text { wine }\end{array}$} \\
\hline & Fermentation & & \\
\hline & $\begin{array}{l}\text { Post-fermentation, maturation and } \\
\text { aging }\end{array}$ & & \\
\hline & $\begin{array}{l}\text { Related activities: } \\
\text {-Equipment preparation } \\
\text { - Guard cellar }\end{array}$ & & \\
\hline \multirow{3}{*}{$\begin{array}{l}\text { Disposal of } \\
\text { wine }\end{array}$} & Mixing different varieties of wine & \multirow{3}{*}{$\begin{array}{l}\text { Direct costs: the cost of wines } \\
\text { blended, hourly rate of direct workers, } \\
\text { the cost of equipment (depreciation, } \\
\text { maintenance, energy consumption) the } \\
\text { cost of auxiliary materials }\end{array}$} & \multirow[b]{3}{*}{ No. of bottles } \\
\hline & Clarification and stabilization & & \\
\hline & $\begin{array}{l}\text { Preparing for sale (bottling, } \\
\text { corking, labeling) }\end{array}$ & & \\
\hline
\end{tabular}

Source: own systematization 
For the first activity, we have the following equation for determining the cost of raw materials:

Cost of raw materials $1=$ Cost of grapes + Proportionate costs related to the activities performed for collecting grapes

For other activities, the relationship will be one recurrent:

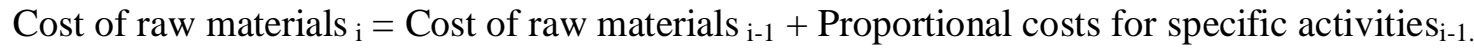

After allocating direct and indirect costs on the activities identified in the $\mathrm{ABC}$ method has resulted the following situation (Table no. 4):

Table no.4.

Direct cost allocation on activities according to $\mathrm{ABC}$ method

\begin{tabular}{|c|c|c|c|c|c|}
\hline \multicolumn{6}{|c|}{ P1. Grape reception } \\
\hline A 1.1 & Collection/purchase raw materials & grapes & wag & 365.8 & 2926400 \\
\hline \multirow[t]{4}{*}{ A 1.2} & $\begin{array}{l}\text { Grape reception: } \\
\text { - Testing the quality } \\
\text { - weighing }\end{array}$ & Total manual work: & & & 2898.54 \\
\hline & $\begin{array}{l}\text { Grape reception: } \\
\text { - Testing the quality } \\
\text { - weighing }\end{array}$ & Direct revenues & wag & 365.8 & 860.68 \\
\hline & $\begin{array}{l}\text { Grape reception: } \\
\text { - Testing the quality } \\
\text { - weighing }\end{array}$ & power auger & wag & 365.8 & $2,037.86$ \\
\hline & & Total materials of which: & & & 194.3 \\
\hline \multirow[t]{5}{*}{ A 1.3} & Related activities & shovels & units & 10 & 24.3 \\
\hline & & buckets & units & 40 & 48.6 \\
\hline & & straw brushes & units & 50 & 60.7 \\
\hline & & mature & units & 100 & 60.7 \\
\hline & Total direct costs $\mathrm{P} 1$ & & wag & 365.8 & 2929492.84 \\
\hline
\end{tabular}

\begin{tabular}{|c|c|c|c|c|c|}
\hline \multicolumn{6}{|c|}{ P2. Wine production } \\
\hline \multirow[t]{5}{*}{ A 2.1} & \multirow{2}{*}{$\begin{array}{l}\text { Pre fermentation activities } \\
\text { - destemming }\end{array}$} & \multirow{2}{*}{$\begin{array}{l}\text { Raw materials: } \\
\text { - grapes }\end{array}$} & \multirow[b]{2}{*}{ wag } & \multirow[b]{2}{*}{365.8} & \multirow[t]{2}{*}{2929492.84} \\
\hline & & & & & \\
\hline & - crushing & Total manual labour: & & & 480.58 \\
\hline & - pumping in fermentation & power auger & wag & 365.8 & $2,037.86$ \\
\hline & tanks & maintenance bunches & wag & 365.8 & $1,272.81$ \\
\hline & & Directing pomace by pressing & wag & 135.3 & 79.33 \\
\hline & & Serving presses & wag & 135.3 & 318.35 \\
\hline & & Placing the tape marc & wag & 58.5 & 130.00 \\
\hline & & Sulfitation grapes + must & wag & 365.8 & 898.91 \\
\hline & & Serving pumps mash decanted & wag & 65.9 & 40.78 \\
\hline & & Transferring burble decantation & wag & 54.9 & 101.18 \\
\hline & & Transfuse mash oversulfitation & wag & 11 & 20.27 \\
\hline & & Maintain daily hygiene & wag & 850 & 934.47 \\
\hline & & Total materials of which: & & & $\mathbf{5 3 1 7 . 0 2}$ \\
\hline & & Bulbs & units & 50 & 14 \\
\hline & & Rivets & $\mathrm{kg}$ & 45 & 56.1 \\
\hline & & Sulphur & $\mathrm{kg}$ & 200 & 59.4 \\
\hline & & Rush & $\mathrm{kg}$ & 400 & 93.4 \\
\hline & & Paraffin & $\mathrm{kg}$ & 500 & 336 \\
\hline & & Calcined Soda & $\mathrm{kg}$ & 1000 & 179 \\
\hline & & Bentonite & $\mathrm{kg}$ & 8000 & 2990 \\
\hline & & Gauze & $\mathrm{ml}$ & 30 & 1.12 \\
\hline & & Oak staves & $\mathrm{mc}$ & 10 & 1121 \\
\hline & & Checker plate & $\mathrm{kg}$ & 500 & 467 \\
\hline
\end{tabular}




\begin{tabular}{|c|c|c|c|c|c|}
\hline & Total direct costs A2.1 & & & & 2935290.44 \\
\hline \multirow[t]{19}{*}{ A 2.2} & Fermentation & Raw materials: & & & \\
\hline & & - mash & & & 2935290.44 \\
\hline & & Total manual labour: & & & 4527.22 \\
\hline & & Transferring mash oversulfitation & wag & 11 & 25.88 \\
\hline & & $\begin{array}{l}\text { Supervised installation concentrated } \\
\text { mash }\end{array}$ & wag & 274.5 & 432.21 \\
\hline & & $\begin{array}{l}\text { Quantitative and qualitative } \\
\text { reception concentrated mash }\end{array}$ & wag & 274.5 & 125.67 \\
\hline & & Transferring concentrate mash & wag & 274.5 & 505.91 \\
\hline & & Conditioning dishes & wag & 530 & $1,165.34$ \\
\hline & & Maintain daily hygiene & wag & 800 & 879.5 \\
\hline & & Prepared SO2 solution & wag & 450 & 247.36 \\
\hline & & Prepared dishes for wine & wag & 450 & 989.44 \\
\hline & & Prepared wine inventory & wag & 450 & 6.8 \\
\hline & & Prepared spaces for wine & wag & 450 & 989.44 \\
\hline & & Transport equipment and mounted & wag & 450 & 765 \\
\hline & & Transferring after fermentation & wag & 274.5 & 754.45 \\
\hline & & Warehouse evacuation & wag & 27.5 & 45.35 \\
\hline & & Washing dishes & wag & 274.5 & 452.67 \\
\hline & & Transfuse yeast & to & 274.5 & 905.34 \\
\hline & Total direct costs A2.2 & & & & 2939817.66 \\
\hline \multirow[t]{29}{*}{ A 2.3. } & Post fermentation & Raw materials: & & & 2939817.66 \\
\hline & Maturation and aging & Total manual labour: & & & 4155.15 \\
\hline & & Preparing Sulphur filters & wag & 247.1 & 351.55 \\
\hline & & Making full and SO2 correction & wag & 247.1 & 607.22 \\
\hline & & Wine treatment with bentonite & wag & 247.1 & 351.55 \\
\hline & & Sampling Laboratory & wag & 247.1 & 581.4 \\
\hline & & Maintain daily hygiene & wag & 225 & 247.36 \\
\hline & & Making full and accurate $\mathrm{SO} 2$ & wag & 274.5 & 169.87 \\
\hline & & Laboratory samples & wag & 248.7 & 273.41 \\
\hline & & Transferring decanting & wag & 274.5 & 606.98 \\
\hline & & Warehouse evacuation & to & 274.5 & 60.47 \\
\hline & & Washing dishes & wag & 274.5 & 603.56 \\
\hline & & Transfuse yeast & to & 274.5 & 301.78 \\
\hline & & Total materials of which: & & & 8152.34 \\
\hline & & Lamps & units & & 280.34 \\
\hline & & Wire brush & units & & 124 \\
\hline & & Insulating gloves & units & & 56 \\
\hline & & Fir wood & $\mathrm{mc}$ & & $1,240.00$ \\
\hline & & Pine ball & $\mathrm{mc}$ & & 765 \\
\hline & & Limestone & $\mathrm{kg}$ & & 125 \\
\hline & & Paint & $\mathrm{kg}$ & & 676 \\
\hline & & Cement & units & & 345 \\
\hline & & Galvanized sheet & $\mathrm{kg}$ & & 512 \\
\hline & & Other materials & $\mathrm{kg}$ & & 2256 \\
\hline & & Coveralls & per & & 1298 \\
\hline & & PSI hose & $\mathrm{ml}$ & & 54 \\
\hline & & Rubber boots & per & & 421 \\
\hline & & Electricity & $\mathrm{kw}$ & 1000 & 1100 \\
\hline & \multicolumn{4}{|l|}{ Total direct costs A2.3 } & 2953225.15 \\
\hline A 2.4 . & Related activities & Guard cellar & hours & 2110 & 1200 \\
\hline & \multicolumn{2}{|c|}{ Total direct costs A 2.1-A 2.4 } & & & 2954425.15 \\
\hline
\end{tabular}




\begin{tabular}{|c|c|c|c|c|c|}
\hline \multicolumn{6}{|c|}{ P3. Disposal of wine } \\
\hline \multirow[t]{7}{*}{ A3.1. } & \multirow{2}{*}{$\begin{array}{l}\text { Blending } \\
\text { fermentating wines } \\
\end{array}$} & Raw materials: & & & 2954425.15 \\
\hline & & Lucrări manuale total: & & & 497.01 \\
\hline & & Quality wine reception & Wag. & 290 & 61.89 \\
\hline & & Medium samples for laboratory & $\mathrm{t}$ & 290 & 95.11 \\
\hline & & Unloading wine & $\mathrm{t}$ & 290 & 178.16 \\
\hline & & Blending wines & $\mathrm{t}$ & 290 & 161.85 \\
\hline & Total A 3.1 & & & & 2954922.16 \\
\hline \multirow[t]{38}{*}{ A3.2. } & $\begin{array}{l}\text { Clasification and } \\
\text { stabilization }\end{array}$ & Raw materials: & & & 2954922.16 \\
\hline & & Total manual labour: & & & 4935.67 \\
\hline & & Wine treatment with bentonite & $\mathrm{t}$ & 290 & 206.29 \\
\hline & & Homogenization of wine & $\mathrm{t}$ & 290 & 226.92 \\
\hline & & Pulling wine on deposit & $\mathrm{t}$ & 290 & 142.53 \\
\hline & & evacuation warehouse & $\mathrm{t}$ & 290 & 127.53 \\
\hline & & Treatment of wine with tannin and gels & $\mathrm{t}$ & 290 & 206.29 \\
\hline & & Mixing and blending wine & $\mathrm{t}$ & 290 & 185.67 \\
\hline & & Dragging wine on the lees & $\mathrm{t}$ & 290 & 142.53 \\
\hline & & Subjecting the wine to heat shock & $\mathrm{t}$ & 290 & 742.65 \\
\hline & & Wine filtration for work & $\mathrm{t}$ & 290 & 618.88 \\
\hline & & Dragging wine in pressure dishes & $\mathrm{t}$ & 290 & 168.79 \\
\hline & & Making fullness & $\mathrm{t}$ & 290 & 79.70 \\
\hline & & Making decanting & $\mathrm{t}$ & 290 & 142.53 \\
\hline & & Correction alcohol blends MC & $\mathrm{t}$ & 290 & $1,155.65$ \\
\hline & & Correction blends sorbic acid SO2 & $\mathrm{t}$ & 290 & $1,155.65$ \\
\hline & & Reception concentrated mash & & 290 & 57.84 \\
\hline & & Transferring dispatch yeasts & $\mathrm{t}$ & 14.5 & 47.82 \\
\hline & & Conditioning dishes & $\mathrm{t}$ & 530 & $1,165.34$ \\
\hline & & Prepared $\mathrm{SO} 2$ solution & $\mathrm{t}$ & 450 & 247.36 \\
\hline & & Sulphite of wines with $\mathrm{SO} 2$ & $\mathrm{t}$ & 365.8 & $1,005.38$ \\
\hline & & Energy: & & & $24,274.80$ \\
\hline & & Pumps & $\mathrm{kw}$ & 90 & $7,499.94$ \\
\hline & & Ventilation & $\mathrm{kw}$ & 201.3 & $16,774.86$ \\
\hline & & Total materials of which: & & & $34,213.28$ \\
\hline & & Particle filter & units & 30 & $4,680.90$ \\
\hline & & EC sterile plates & units & 30 & $20,163.90$ \\
\hline & & Sulphur dust & $\mathrm{kg}$ & 0.6 & 5.4 \\
\hline & & Trisodium phosphate & $\mathrm{kg}$ & 0.03 & 3.6 \\
\hline & & Calcined soda & $\mathrm{kg}$ & 0.03 & 4.86 \\
\hline & & Bentonite & $\mathrm{kg}$ & 10 & 286.86 \\
\hline & & Gelatin & $\mathrm{kg}$ & 0.05 & 28.51 \\
\hline & & Citric acid & $\mathrm{kg}$ & 5 & $1,500.29$ \\
\hline & & Sorbic acid & $\mathrm{kg}$ & 2 & $6,601.28$ \\
\hline & & Ferrocyanide K & $\mathrm{kg}$ & 0.15 & 58.51 \\
\hline & & Bicarbonate $\mathrm{K}$ & $\mathrm{kg}$ & 1.5 & 801.15 \\
\hline & & $\mathrm{SO} 2$ & $\mathrm{~kg}$ & 1 & 78.02 \\
\hline & Total A 3.2 & & & & $\mathbf{3 , 0 1 8 , 3 4 5 . 9 1}$ \\
\hline \multirow[t]{10}{*}{ A3.3. } & Prepairing for sale & Raw materials: & & & 3018345.91 \\
\hline & & Total manual labour: & & & 33193.39 \\
\hline & - botteling & Salaries foreman and shift boss & & & 2376.88 \\
\hline & - Sealing & Powered empty bottles tape & $\mathrm{mb}$ & 2500 & 417.33 \\
\hline & - Labeling & $\begin{array}{l}\text { Powered dishwasher with detergent and lye } \\
\text { water }\end{array}$ & $\mathrm{mb}$ & 2500 & 378.23 \\
\hline & & Mechanical washing bottles & $\mathrm{mb}$ & 2500 & 575.63 \\
\hline & & Control bottles washed & $\mathrm{mb}$ & 2500 & 546.68 \\
\hline & & Filled bottles of wine mechanically & $\mathrm{mb}$ & 2500 & 1604.53 \\
\hline & & Control full bottles & $\mathrm{mb}$ & 2500 & 546.68 \\
\hline & & Returned bottles with impurities & $\mathrm{mb}$ & 2500 & 521.72 \\
\hline
\end{tabular}




\begin{tabular}{|c|c|c|c|c|}
\hline \multicolumn{5}{|l|}{ P3. Disposal of wine } \\
\hline & Stapled or mechanical sealing & $\mathrm{mb}$ & 2500 & 575.63 \\
\hline & Applied thermal hoods & $\mathrm{mb}$ & 2500 & 518.36 \\
\hline & Labelled mechanically & $\mathrm{mb}$ & 2500 & 758.1 \\
\hline & Applied flyers & $\mathrm{mb}$ & 2500 & 575.63 \\
\hline & Leveling bottles containing & $\mathrm{mb}$ & 2500 & 758.1 \\
\hline & Control bottles aesthetic & $\mathrm{mb}$ & 2500 & 758.1 \\
\hline & Control CTC - end band & $\mathrm{mb}$ & 2500 & 546.73 \\
\hline & Retrieved bottles from band - sitting shuttles & $\mathrm{mb}$ & 2500 & 1555.07 \\
\hline & Sort shuttle & $\mathrm{mb}$ & 2500 & 417.33 \\
\hline & Transport crates and pallets broken & $\mathrm{mb}$ & 2500 & 417.33 \\
\hline & Sitting empty crates on tape - received & $\mathrm{mb}$ & 2500 & 575.63 \\
\hline & Washed and sanitized with softened water & $\mathrm{mb}$ & 2500 & 539.24 \\
\hline & $\begin{array}{l}\begin{array}{l}\text { Washed and sanitized bottling plant } \\
\text { sterilization }\end{array} \\
\end{array}$ & $\mathrm{mb}$ & 2500 & 539.24 \\
\hline & Filtered wine (filter format - removed cards) & $\mathrm{mb}$ & 2500 & 523.38 \\
\hline & Pasteurized wine & $\mathrm{mb}$ & 2500 & 628.05 \\
\hline & Sort and label dating & $\mathrm{mb}$ & 2500 & 518.69 \\
\hline & $\begin{array}{l}\text { Quantitative and qualitative reception of } \\
\text { bottles }\end{array}$ & $\mathrm{mb}$ & 2500 & 582.86 \\
\hline & Shards removed and taken to the container & $\mathrm{mb}$ & 2500 & 7.56 \\
\hline & Hygiene Washing Filling & $\mathrm{mb}$ & 2500 & 518.36 \\
\hline & Purchasing materials & $\mathrm{mb}$ & 2500 & 417.33 \\
\hline & Placing of bottles in cardboard & $\mathrm{mb}$ & 2500 & 758.10 \\
\hline & Secured and sealed boxes & $\mathrm{mb}$ & 2500 & 518.36 \\
\hline & Placing boxes on conveyor belt & $\mathrm{mb}$ & 2500 & 1236.85 \\
\hline & Handling containers filled & $\mathrm{mb}$ & 2500 & 4785.58 \\
\hline & Handling of empty packaging & $\mathrm{mb}$ & 2500 & 4785.58 \\
\hline & Plugs removed manually & $\mathrm{mb}$ & 2500 & 46.96 \\
\hline & Stained glasses manually washed & $\mathrm{mb}$ & 2500 & 352.22 \\
\hline & Prepairing and closing activities & $\mathrm{mb}$ & 2500 & 758.1 \\
\hline & Energy and water: & & & 16490.63 \\
\hline & Energy & $\mathrm{kw}$ & 150000 & $16,490.63$ \\
\hline & Total materials of which: & & & 123781.07 \\
\hline & Spare parts & & & 5002.16 \\
\hline & mature pvc & pieces & 30 & 21.87 \\
\hline & Brush for washing glasses & units & 100 & 36.44 \\
\hline & Brushes PVC & units & 20 & 10.93 \\
\hline & Lighting lamps & units & 10 & 11.66 \\
\hline & Different paint & 1 & 100 & 388.74 \\
\hline & Caustic Soda & $\mathrm{Kg}$ & 7000 & $3,061.32$ \\
\hline & Calcined Soda & $\mathrm{Kg}$ & 3500 & $1,020.44$ \\
\hline & Trisodium phosphate & $\mathrm{kg}$ & 3000 & $1,749.33$ \\
\hline & metal staples & units & 2000000 & $9,146.80$ \\
\hline & Cork stoppers & units & 320000 & $14,955.02$ \\
\hline & Thermal hoods & units & 680000 & $7,094.49$ \\
\hline & Particle filter & units & 16000 & $28,583.76$ \\
\hline & carton boxes & units & 25000 & $11,433.50$ \\
\hline & Role gelux & units & 800 & 291.55 \\
\hline & Wine labels 0,75 & units & 310000 & $1,196.23$ \\
\hline & Wine labels 1/1 & units & 1930000 & $7,447.50$ \\
\hline & Bands & units & 2230000 & $18,141.89$ \\
\hline & Stack PVC & & 500 & 824.53 \\
\hline & Bottles 1/1 & & 70000 & $11,004.75$ \\
\hline & Bottles 0,75 & & 15000 & $2,358.16$ \\
\hline Total A3.3 & & & & $3,191,811.00$ \\
\hline Total P3 & & & & 3191811 \\
\hline
\end{tabular}

Source: own calculations 
For calculating the unit cost on activities and on products, it is necessary the quantitative determination of the cost drivers, which is made based on technology of production records, and accounting data (Table no. 5.)

Table no. 5 .

Calculation of unit cost of production by the ABC method for assortment Italian Riesling

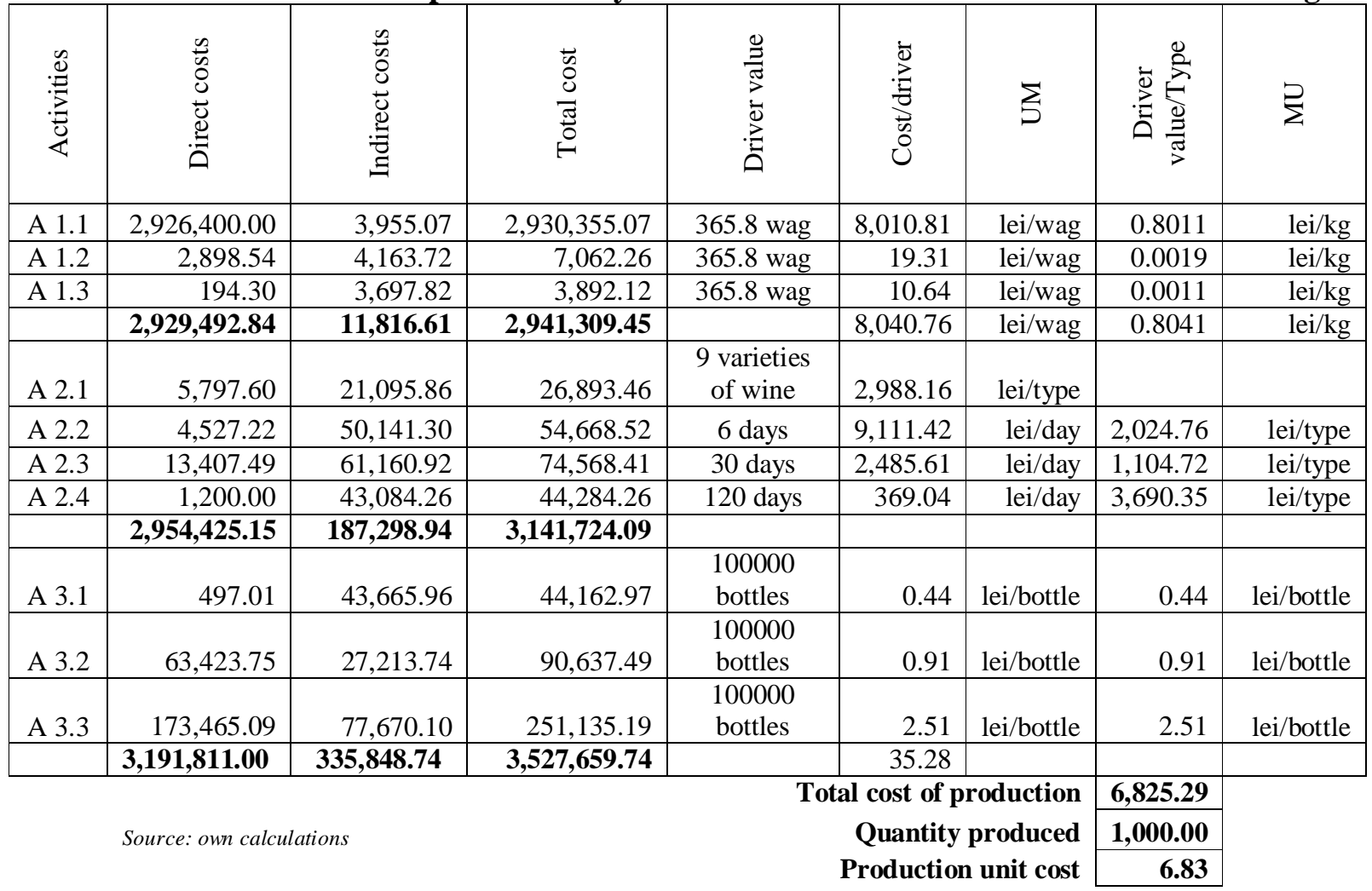

\section{Balanced Scorecard}

Starting from the statement of Lord Kelvin who states that you can not improve what you do not measure, Norton and Kaplan in 1992 introduced the concept of Balanced Scorecard (BS) based on the results of a study by Nolan and Norton on measuring the performance of companies whose intangible assets play a central role in value creation. The result of the study was to obtain a set of indicators that can give managers a fast but comprehensive view on the performance of the entity. At the same time, the authors hypothesized that tracking performance using this holistic approach will increase the likelihood that organizations achieve their goals; this hypothesis was confirmed by studies conducted in the last twenty years (Chenhall and Langfield-Smitth, 2007). Due to the success of the method applyed by a large number of companies, limited to intangible assets initial concept was extended to the necessary management tool description, communication and implementation strategies (Kaplan, 2010). According to the authors, the method Balanced Scorecard is defined as a management system that enables organizations to clarify their vision and strategy and transform them into actions (Kaplan and Norton, 1996). According to another definition Balanced Scorecard method is a multidimensional approach to performance measurement and management, strictly related to the organizational strategy (Otley, 1999). Experience has shown that measuring the performance of an organization is a process without which you can not define a management strategy, the utility method BS being so obvious. 
By offering performance indicators is provided both useful information to decision making process and a basis for many activities such as (Kaplan and Norton, 1996):

- Strategic feedback to those involved in decision making about the current state of the organization of the four perspectives;

- Diagnostic feedback provided to a variety of processes in order to target improvements in progress;

- Identification of performance trends over time;

- Providing quantitative inputs in the forecasting models and decision support used.

Performance indicators should reflect the most important results of the entity (relevance) they must be easily calculated from the available data in various departments (measurability), to correlate the results of all entity levels (integration capacity) to have a clear formula of calculation and its foundation so that various approaches to lead to the same calculation modality (reliability). Also, the indicator system must be able to provide an estimate of the trend of future results (sustenability), the results provided must be managed, and how they are formulated must be easily explained to the stakeholders (communicability). It is also important to ensure the consistency of indicator system, a way to control the possibility of manipulation of results (reliability) as well as a large enough variety of indicators that can provide a clear and detailed picture to the personnel on specific objectives achieved (differentiation).

Balanced Scorecard turn the vision and strategy of an economic entity into objectives and performance measures grouped into four areas: learning and development, internal processes, financial and customer satisfaction, which are key indicators in identifying causal relationships between them. Organizational learning and development perspective examines the changes and improvements needed by the economic entity in order to turn the vision into reality. The perspective of the internal processes describes the identification of the main business processes which must be applied to respond to the needs of the customers and the shareholders. In other words the main process should identify indicators that the economic entity wants to control who will be part of balanced scorecard. The financial perspective helps to identify the image that entity want to show to the shareholders, while the clients perspective helps establish the image that the economic entity adopts to the clients. Synthetic on steps go like this:

Step 1. Defining the vision, mission and strategy

The success of the economic entity can be achieved by managing the rapid changes in the evolution of the wine industry from a fragmented farming industry towards a professional industry as well as the modification of the clients needs through continous adapting to inovations at the market demand and at its complexity as well as consumers needs.

The mision of the company is: to achive a market share which is enough for the company to be the leader at the national level, as well as positioning in the top ten European wine producers; strengthening its position in the local market by offering quality wines and expand the distribution network in especially exports; sustainable growth of the business both in terms of turnover and maximize shareholder value.

The company's strategy is to: expand the distribution network, introduction of new types of wine in production; maintenance and strengthening the position owned on the market of wine producers; fast adaptability to local and international market of wine; reducing dependency on certain markets or groups of products. The main values of the company include: focusing on consumer requirements; customer loyalty; focus on quality and sustainability of the business; maintaining a friendly climate in relations with employees; business practice respecting ethical values of honesty and integrity.

Step 2. The development of operational objectives that characterize the performance, indicators to measure their achievement, including the targets 
The creation of performance measures was taken to ensure the link between them and the strategic vision of the organization. Also, to each object defined in the four perspectives was associated at least one performance indicator associated with that object characterization. Indicators were defined exactly, including object of calculation, method of quantification, the data and the time period under evaluation (Tables 6-9). In defining or choosing indicators, it was intended to include both quantitative quantities and qualitative. Even if quantitative sizes provide objective information that can serve to justify management decisions related to resource allocation, quality sizes are also important, even if they have a high degree of subjectivity being based on the experiences of customers, managers and employees. What is crucial, however, is to ensure a balance between qualitative and quantitative indicators.

Table no. 6 .

The financial dimension

\begin{tabular}{|c|c|c|c|}
\hline Objectives & Indicators & Targets & Action / Initiatives \\
\hline $\begin{array}{l}\text { Maintaining a certain } \\
\text { level of current liquidity } \\
\text { to ensure normal activity }\end{array}$ & Cash flows & $\begin{array}{l}\text { - Maintaining at a } \\
\text { constant level cash flows }\end{array}$ & $\begin{array}{l}\text { - Monitor the collection } \\
\text { of receivables }\end{array}$ \\
\hline $\begin{array}{l}\text { Keeping turnover to a } \\
\text { level at least equal to that } \\
\text { of the previous year }\end{array}$ & $\begin{array}{l}\text { Turnover } \\
\text { Variation of turnover }\end{array}$ & $\begin{array}{l}\text { - Increased turnover by } \\
5 \% \text { from the previous } \\
\text { year }\end{array}$ & $\begin{array}{l}\text { - twice a month analysis } \\
\text { of the turnover }\end{array}$ \\
\hline Profit growth & $\begin{array}{l}\text { Market share } \\
\text { Financial rate: ROI, ROE }\end{array}$ & $\begin{array}{l}\text { - increasing market share } \\
\text { with } 10 \% \\
\text { - The existence of } \\
\text { positive changes in the } \\
\text { value of financial ratios } \\
\text { calculated in relation to } \\
\text { the previous year }\end{array}$ & $\begin{array}{l}\text { - Monthly analysis of the } \\
\text { market share } \\
\text { - Monthly calculation of } \\
\text { financial indicators of } \\
\text { efficiency }\end{array}$ \\
\hline
\end{tabular}

Source: author's own vision

Table no. 7.

Dimension customers

\begin{tabular}{|l|l|l|l|}
\hline \multicolumn{1}{|c|}{ Objectives } & \multicolumn{1}{|c|}{ Indicators } & \multicolumn{1}{c|}{ Targets } & \multicolumn{1}{c|}{ Action / Initiatives } \\
\hline $\begin{array}{l}\text { Developing new type of } \\
\text { wine }\end{array}$ & $\begin{array}{l}\text { Percentage of new } \\
\text { varieties of wine sales in } \\
\text { total sales } \\
\text { Report new varieties / } \\
\text { varieties existing }\end{array}$ & $\begin{array}{l}\text {-indicator value 5\% } \\
\text {-growth of new products } \\
\text { by 10\% over the previous } \\
\text { year }\end{array}$ & $\begin{array}{l}\text {-monitoring research and } \\
\text { development activity } \\
\text { - Continuous analysis of } \\
\text { the market, especially the } \\
\text { main competitors }\end{array}$ \\
\hline $\begin{array}{l}\text { Developing partnerships } \\
\text { with major customers } \\
\text { of regular customers }\end{array}$ & $\begin{array}{l}\text { Variation of the number } \\
\text { regular customers } \\
\text {-attracting a number of 4 } \\
\text { customers belonging to } \\
\text { the international market }\end{array}$ & $\begin{array}{l}\text {-increased by 20\% of } \\
\text { domestic and foreign } \\
\text { market } \\
\text { - Expansion of } \\
\text { distribution channels } \\
\text { - Improve the efficiency } \\
\text { of sales department }\end{array}$ \\
\hline $\begin{array}{l}\text { Ensuring continuous } \\
\text { distributions }\end{array}$ & $\begin{array}{l}\text { On time distribution of } \\
\text { products (valued by } \\
\text { customer) }\end{array}$ & $\begin{array}{l}- \text { Achieve a minimum } \\
\text { score of 4 provided by } \\
\text { customers (on a scale of } \\
1-5) \text { for rapid fulfilling } \\
\text { orders }\end{array}$ & $\begin{array}{l}\text { - Preparing and } \\
\text { completing questionnaires } \\
\text { by major customers }\end{array}$ \\
\hline $\begin{array}{l}\text { Maintaining customer } \\
\text { loyalty }\end{array}$ & $\begin{array}{l}\text { Sales situation on } \\
\text { customers and periods } \\
\text { client older than one year }\end{array}$ & $\begin{array}{l}\text { - Preparation of sales } \\
\text { reports twice a month and } \\
\text { analysis of their dynamics }\end{array}$ \\
\hline
\end{tabular}

Source: author's own vision 
Table no. 8 .

Internal processes

\begin{tabular}{|l|l|l|l|}
\hline \multicolumn{1}{|c|}{ Objectives } & \multicolumn{1}{c|}{ Indicators } & \multicolumn{1}{c|}{ Targets } & \multicolumn{1}{c|}{ Action / Initiatives } \\
\hline $\begin{array}{l}\text { Improving technology } \\
\text { lines }\end{array}$ & $\begin{array}{l}\text { The value of investments } \\
\text { compared to the turnover }\end{array}$ & $\begin{array}{l}\text { - Increased investment } \\
\text { compared to the previous } \\
\text { year by at least 10\% }\end{array}$ & $\begin{array}{l}\text { - Analysis of investment } \\
\text { needs }\end{array}$ \\
\hline $\begin{array}{l}\text { Efficiency of } \\
\text { technological processes }\end{array}$ & $\begin{array}{l}\text { The time value of } \\
\text { production cycle on } \\
\text { varieties of wine } \\
\text { The unit cost of each } \\
\text { wine variety } \\
\text { Operating profit on wine } \\
\text { varieties }\end{array}$ & $\begin{array}{l}\text { - Reduction of the } \\
\text { production cycle } \\
\text { - Lowering the cost of } \\
\text { production by 5\% } \\
\text { - Operating profit } \\
\text { increased by 5\% }\end{array}$ & $\begin{array}{l}\text { - Updating manufacturing } \\
\text { lines } \\
\text { - Analysis of production } \\
\text { costs by ABC method } \\
\text { - Analyze monthly } \\
\text { operating profit by } \\
\text { categories of activity and } \\
\text { products and to identify } \\
\text { areas that can be } \\
\text { improved }\end{array}$ \\
\hline Products design & $\begin{array}{l}\text { Number of modifications } \\
\text { brought to the labels }\end{array}$ & $\begin{array}{l}\text { - Change of at least 25\% } \\
\text { of the labels }\end{array}$ & $\begin{array}{l}\text { - the existence of a } \\
\text { permanent concern for } \\
\text { product design }\end{array}$ \\
\hline Developing new products & $\begin{array}{l}\text { Report new products / } \\
\text { existing products }\end{array}$ & $\begin{array}{l}\text { - Introducing a minimum } \\
\text { of 2 new products }\end{array}$ & $\begin{array}{l}\text { - Monitoring the progress } \\
\text { of the development of } \\
\text { new products } \\
\text { - Continuous analysis of } \\
\text { market competitors }\end{array}$ \\
\hline
\end{tabular}

Source: author's own vision

Table no. 9.

Growth and Learning

\begin{tabular}{|l|l|l|l|}
\hline \multicolumn{1}{|c|}{ Objectives } & \multicolumn{1}{|c|}{ Indicators } & \multicolumn{1}{c|}{ Targets } & \multicolumn{1}{c|}{ Action / Initiatives } \\
\hline $\begin{array}{l}\text { Increasing staff } \\
\text { qualification and }\end{array}$ & $\begin{array}{l}\text { Number of trainings / No. } \\
\text { employees } \\
\text { Percentage of employees } \\
\text { trained regularly }\end{array}$ & $\begin{array}{l}\text { - Increased by 15\% from } \\
\text { the previous year of } \\
\text { trainings conducted } \\
-40 \% \text { increase in the } \\
\text { number of employees } \\
\text { participating in training } \\
\text { activities }\end{array}$ & $\begin{array}{l}\text { - Develop a plan for } \\
\text { human resource } \\
\text { development by } \\
\text { specialized department } \\
\text { - Identify training needs } \\
\text { of staff } \\
\text { - Monitoring the } \\
\text { performance of } \\
\text { employees participating } \\
\text { in training activities }\end{array}$ \\
\hline $\begin{array}{l}\text { Focusing on product } \\
\text { quality }\end{array}$ & $\begin{array}{l}\text { No. Scrap / production } \\
\text { value }\end{array}$ & $\begin{array}{l}- \text { Weekly analysis of the } \\
\text { number of rejects } \\
\text { Decrease waste by at least } \\
10 \% \text { over the previous } \\
\text { year }\end{array}$ & $\begin{array}{l}\text { - Identify the causes of } \\
\text { the existence of scrap }\end{array}$ \\
\hline $\begin{array}{l}\text { Introduction of new } \\
\text { products }\end{array}$ & $\begin{array}{l}\text { Number of products } \\
\text { introduced / No products } \\
\text { entered by the main } \\
\text { competitor }\end{array}$ & $\begin{array}{l}\text { - introduction of at least } \\
\text { two new products }\end{array}$ & $\begin{array}{l}\text { - Continuous analysis of } \\
\text { market } \\
\text { - Improve the efficiency } \\
\text { of research and } \\
\text { development department } \\
\text { - Identify potential market } \\
\text { opportunities for niche } \\
\text { products }\end{array}$ \\
\hline
\end{tabular}

Source: author's own vision 


\section{Remarks based on the cases presented and analyzed}

The most important step in the modeling phase of the ABC system is gathering information on the needs of users of the system (which are key decisions that they need to develop and that is the frequency with which this work is done, or what information about their costs are needed in decision-making) to be made in detail, through direct talks made between a member of the implementation team and each user, and by filling in questionnaires.

Another very important element is the creation of ABC model that identify resources, drivers, activity centers, cost drivers and cost items and cost benchmarks. Transposition activity of the operations and processes performed within an entity is complex and requires time to analyze the information and identify the key ones (as we noted above, the level of detail of activities is an important factor in the implementation of the ABC method - a too much detailing can consume too many resources compared to the results provided, as a superficial detailing may not provide information relevant to the decision-making process). But the most important step in developing the model is to identify resources and cost drivers which must be chosen so as to reflect a cause and effect relationship. A correct identification of subsequently allow simple allocation of resources to activities and then of activities on products.

Balanced Scorecard incorporates causal relationships with impact on strategic objectives, allowing periodic assessment of critical success factors and making adjustments if necessary. Knowing in detail the causal relationships by analyzing dashboard managers observed deviations from targets as soon as they occur and can act quickly on the factors that produced them (BoțaAvram, 2012).

Another benefit of applying the ABC method is to use the data provided by it in applying Balanced Scorecard method, the second part of the empirical study showed how the two methods can be integrated. Purpose of implementing the Balanced Scorecard method consisted in: facilitating the achievement of the company's mission and objectives set, aligning the company's existing standards work to the wine industry standards, ensuring consistency between existing activities redefined in the context of strategic management approach, providing a tool to evaluate the activity and measure the progress.

\section{Conclusions}

In the implementation of the $\mathrm{ABC}$ method there are a series of previous activities effective implementation of the method, which should be given particular importance because it influences directly the success of its implementation and objectives set, such as defining form of ABC system, continuous training of both the implementation team members and personnel, continue gathering of information developing a general or permanent revision of the method performance.

To properly reflect the vision of the company's staff in the design and implementation of method, it is necessary to form a team comprising representatives of the departments of production, accounting, sales, IT and R \& D, and to have very good and detailed knowledge of all operations performed in their departments and also personal qualities that facilitate a good communication of the results they obtain to other employees as well as knowledge transfer.

A series of important decisions need to be developed in the design phase of the model when it is important to determine if it will be an independent or an integrated system in the existing management accounting system, the degree of accuracy or detail of the work, how they wish to elaborate reporting, and its complexity. Relative to the training the parties involved in the the implementation of the ABC system it should be targeted three levels of staff: senior management, the implementation team and the users of the system, which is required to be given training differentiated according to their specific needs.

As can be seen from the analysis of the elements contained in the four dimensions designed to implement the Balanced Scorecard method, the method concerns the company's future, and what it wants to achieve. The central point of the method refers to the strategy and vision, and not to 
exercise excessive control and central premise is that employees will adopt the necessary behaviors and will exercise responsibilities to achieve them. Therefore, the definition of concrete measures to assess the four dimensions is important for employees to provide them a basis for self-assessment and subsequent evaluation.

\section{References}

1. Anthony, R. N. \& Dearden, J. D. (1976). Management control systems, $3^{\text {rd }}$ ed., Homewood (Ill.): Irwin.

2. Boța-Avram, C. (2012). Managerial ownership, board structure and firm's performance: a review of main findings, CES Working Papers, vol. IV, Issue 4, December, pp. 648-656.

3. Bouquin H. (2004). Comptabilité de Gestion, Editura Economică, Paris, p.184.

4. Briciu S., (2006). Contabilitate Managerială, Editura Economica, Bucure $\square$ ti.

5. Briciu S., Căpu $\square$ neanu S., Rof L., M., Topor D. (2010). Accounting and management control. Tools for performance evaluation entity, Aeternitas Publishing House, Alba Iulia.

6. Butler, A., Letza, S. R. \& Neale, B. (1997). Linking the balanced scorecard to strategy. Long Range Planning 30, 2, pp. 242-253.

7. Chenhall R., Langfield-Smitth K. (2007), Multiple Perspectives of Performance Measures, European Management Journal, no.25 (4), pp.266-282

8. Chiapello, E. \& Lebas, M. (1996). The Tableau de Bord, a French Approach to Management Information, Communication presented at the19th Annual Meeting of the European Accounting Association, Bergen (Norway), 2-4th May.

9. Demeestère R. (2004). Comptabilité de gestion et mesure des performances, Editura Dunod, Paris, p.34.

10. Ebbeken K., Possler L., Ristea M., (2000). Calculation and management of costs, Teora Publishing House, Bucharest, p. 364.

11. Eccles, R.G. (1991). The Performance Measurement Manifesto. Harvard Business Review 69, 1, pp. 131-37.

12. Epstein, M.J. \& Manzoni, J.F. (1997). The balanced scorecard and tableau de bord: translating strategy into action. Management Accounting (US) 79, 2, pp. 28-36.

13. Frigo, M.L. \& Krumwiede, K.R. (2000). The balanced scorecard. A winning performance measurement system. Strategic Finance 81, 7, pp. 50-54.

14. Groşanu A., Răchişan P.R., Berinde S.-R. (2009). Developing an organization system for management accounting based on responsability centers, Review of Business Research, pp. 50-60.

15. Kaplan R. S. (2010), Conceptual Foundations of the Balanced Scorecard, Working paper 10-074, disponibil la http://www.hbs.edu/research/pdf/10-074.pdf, accesat în data de 09.09.2013.

16. Kaplan, R.S. \& Norton, D.P. (1992). The balanced scorecard-measures that drive performance. Harvard Business Review 70, 1, pp. 61-66.

17. Kaplan, R.S. \& Norton, D.P. (1996). Using the balanced scorecard as a strategic management system. Harvard Business Review 74, 1, pp. 75-85.

18. Kaplan, R.S. \& Norton, D.P. (1997). Why does business need a balances scorecard?. Journal of Cost Management 11, 3, pp. 5-10.

19. Lebas, M. \& Mévellec, J.M. (1999). Vingt ans de chantiers de comptabilité de gestion, Comptabilité, Contrôle, Audit special issue "Les vingt ans de l'AFC", May, pp. 77-91.

20. Lorino, P. (1997). Méthodes et pratiques de la performance - Le guide de pilotage, Les Editions d'Organisation.

21. McNair, C.J., Lynch, R.L., \& Cross, K.L. (1990). Do financial and non-financial measures have to agree? Management Accounting (US) 72, 5, pp. 28-39. 
22. Mendoza, C., \& Zrihen, R. (1999a). Le tableau de bord, en VO ou en version américaine? Revue Française de Comptabilité 309, pp. 60-66.

23. Merchant, K. (1985). Control in Business Organizations. Boston (Ma.): Harvard Graduate School of Business.

24. Miller, J. and Vollman Th. (1985). The Hidden Factory, Harvard Business Review, Vol. 63, No. 5, September/October 1985, p. 142.

25. Otley D. (1999), Performance Management: A Framework for Management Control Systems Research, Management Accounting Research, vol.10, pp. 363-382

26. Parker, L.D. (1979). Divisional performance measurement: beyond an exclusive profit test. Accounting and Business Research 9, pp. 309-319.

27. Porter, M.E., (1985). Competitive Advantage: Creating and Sustaining Superior Performance. New York: Free Press.

28. Răchişan P.R., Groşanu A., Berinde S.-R. (2010). Budgeting system of the economic entities organized by responsability centers, Revista Economica, pp. 242-249.

29. Schoenfeld, H. M. (1986). The Present State of Performance Evaluation in Multinational. In Holzer H.P., \& Schoenfeld H.M. (eds), Managerial Accounting and Analysis in Multinational Enterprises, Berlin: Walter de Gruyter, pp. 217-252.

30. Sharma R. (1998), Management Accounting: Where to Next? Australian CPA, December, pp. 24-25.

31. Tabără N., Briciu S. (Coordonatori) (2012). Actualități şi perspective în contabilitate şi control de gestiune, Editura TipoMoldova, Iaşi, 2012, p.153.

32. Topor D., Căpu $\square$ neanu S., Pu $\square$ an A. (2012) Evolution and Performance analysis for wine Entities in Romania, Annales Universitatis Apulensis Series Oeconomica, Volum 2, Nr. 14, pp 403-412. 